\title{
Impact of the COVID-19 Pandemic on the Quality of Life of Patients with Parkinson's Disease and Their Caregivers: A Single-Center Survey in Tochigi Prefecture
}

Keisuke Suzukia,*, Ayaka Numao ${ }^{\mathrm{a}}$, Tomoko Komagamine ${ }^{\mathrm{a}}$, Yasuo Haruyama ${ }^{\mathrm{b}}$, Akiko Kawasaki ${ }^{\mathrm{a}}$, Kei Funakoshi ${ }^{a}$, Hiroaki Fujita ${ }^{a}$, Shiho Suzuki $^{\mathrm{a}}$, Madoka Okamura ${ }^{\mathrm{a}}$, Tomohiko Shiina $^{\mathrm{a}}$ and Koichi Hirata ${ }^{\mathrm{a}}$

${ }^{a}$ Department of Neurology, Dokkyo Medical University, Tochigi, Japan

${ }^{\mathrm{b}}$ Integrated Research Faculty for Advanced Medical Science, Dokkyo Medical University School of Medicine, Tochigi, Japan

Accepted 3 March 2021

Pre-press 22 March 2021

\begin{abstract}
.
Background: The coronavirus disease 2019 (COVID-19) pandemic has negatively affected the mental health of the general population.

Objective: We investigated the determinants of quality of life (QOL) in Parkinson's disease (PD) patients during the COVID19 pandemic.

Methods: Impacts of lifestyle changes due to the COVID-19 pandemic on 100 patients with PD and their caregivers/spouses were assessed. The Hospital Anxiety and Depression Scale was used to assess anxiety and depression. The physical component summary (PCS) and mental component summary (MCS) scores of the short form (SF)-8 were used to evaluate health-related QOL.

Results: Regarding health-related QOL, physical function, role physical, general health, vitality and the PCS score were significantly worse in PD patients than in caregivers. Worsening of PD-related symptoms, increased stress, and decreased physical activity were observed in $29.0 \%, 37.0 \%$ and $44.0 \%$ of PD patients, respectively. Sixteen patients (16.0\%) experienced problems with hospital access, but none reported medication shortages. Strong concerns about COVID-19 were reported by 47.0\% of caregivers and 50.0\% of PD patients. In PD patients, increased gait disturbance and rigidity, disease severity, smoking, the levodopa equivalent dose and decreased body weight predicted a worse PCS score; anxiety, depression, female sex, stress and long disease duration predicted a worse MCS score. In caregivers, age and smoking contributed to a worse PCS score; depression, stress and worsening patient mood contributed to a worse MCS score.

Conclusion: We report the negative impacts of the COVID-19 pandemic on health-related QOL and its determinants in PD patients and their caregivers.
\end{abstract}

Keywords: Caregivers, COVID-19, Parkinson's disease, quality of life, SARS-CoV-2

\footnotetext{
${ }^{*}$ Correspondence to: Keisuke Suzuki, MD, PhD, Department of Neurology, Dokkyo Medical University, 880 Kitakobayashi, Mibu,
} 


\section{INTRODUCTION}

The novel coronavirus called severe acute respiratory syndrome coronavirus 2 (SARS-CoV-2), which causes coronavirus disease 2019 (COVID-19), was first reported in Wuhan, China, in December 2019 [1] and is responsible for a global pandemic. A nationwide lockdown in Italy during the COVID19 pandemic reportedly negatively impacted mental health, anxiety and sleep [2]. In Japan, a state of emergency was declared on April 7, 2020, in seven prefectures, including Tokyo and Kanagawa; on April 16 until May 31, the state of emergency was expanded throughout Japan to prevent the spread of SARSCoV-2. People were asked to refrain from leaving their homes. Even after the declaration of the state of emergency was lifted, the government instructed the public to continue avoiding 1) closed spaces with poor ventilation, 2) crowded places with many people nearby, and 3) close-contact settings such as closerange conversations, resulting in significant changes in lifestyles and social situations.

Parkinson's disease (PD) is a movement disorder characterized by core motor features, namely, bradykinesia plus rigidity and/or resting tremor, that are caused by the degeneration of nigrostriatal dopaminergic neurons. In addition to dopaminergic dysfunction, other neurotransmitter deficits are common in PD and contribute to various highly prevalent nonmotor features, such as sleep disorders, anxiety, depression, autonomic dysfunction, cognitive impairment, and olfactory impairment [3]. It has been reported that a strong earthquake in Kumamoto Prefecture in Japan resulted in clinical worsening of neuropsychiatric symptoms, such as fear, anxiety, insomnia, and fatigue, and motor symptoms in 335 PD patients who lived in the region [4].

Moreover, a community-based case-control study investigating the direct effect of COVID-19 on PD found that compared with PD patients without COVID-19, PD patients with COVID-19 experienced clinical deterioration of motor and nonmotor symptoms, including cognitive impairment, fatigue and urinary symptoms [5]. Prasad et al. [6] also reported that $11 \%$ of 100 patients with PD in India had worsening motor or nonmotor symptoms during the COVID-19 pandemic but that only $4 \%$ had problems with medical consultations or medication supplies. In contrast, 46 (28\%) of 162 PD patients in Italy experienced an acute exacerbation of clinical symptoms (50\% experienced worsening of their motor symptoms), $52 \%$ of whom required a change in medication
[7]. In a report from Iran, $81.2 \%$ of 137 patients with PD had symptoms of anxiety after the COVID-19 outbreak, with $25.5 \%$ experiencing severe symptoms, and symptoms of anxiety were significantly more common in PD patients than in their caregivers (57.9\% with anxiety and $4.2 \%$ with severe symptoms) [8]. Overall, increased psychological distress during the COVID-19 pandemic may also worsen motor symptoms [9] and various nonmotor symptoms, such as cognitive function [10], anxiety, depression [9] and pain [11], among PD patients. Indeed, in an onlinebased study including 5,429 patients with PD, 43\% and $52 \%$ reported worsened motor and nonmotor symptoms, respectively [12].

The COVID-19 pandemic has impacted the management of patients with neurological disorders. In general, replacing outpatient care with telemedicine, postponing surgery, including deep brain stimulation, and avoiding nonemergency hospitalizations have been recommended for patients with PD and other movement disorders during the COVID-19 pandemic [13]. Therefore, telemedicine has rapidly expanded to provide appropriate medical care [14]. Telemedicine has been introduced in Japan, and it has been particularly promoted for patients who are at risk of severe COVID-19, such as elderly patients and those with underlying diseases. In a study evaluating the clinical profiles of 10 PD patients infected with SARS-CoV-2, older age and a longer duration of PD were associated with increased mortality (40\%), with a $50 \%$ mortality rate in those receiving advanced-stage treatment, such as deep brain stimulation and continuous levodopa infusion [15].

Thus, changes in social conditions during the COVID-19 pandemic may have affected the motor and nonmotor symptoms experienced by PD patients, negatively affecting their quality of life (QOL). However, determinants of the QOL of patients with PD during the COVID-19 pandemic have not been thoroughly studied. Accordingly, we aimed to investigate changes in motor symptoms, cognition, mood, sleep and stress as well as determinants of QOL in PD patients and their spouses/caregivers during the COVID-19 outbreak in Japan in a single-center setting.

\section{METHODS}

We performed a cross-sectional survey between June and December 2020 to assess the impact of changes in social behaviors and lifestyles during 
the COVID-19 pandemic on patients with PD at Dokkyo Medical University Hospital, Tochigi, Japan. Tochigi Prefecture has a population of 2 million and is located in the northern part of Kanto region, which includes the capital of Japan, Tokyo. The institutional review boards of Dokkyo Medical University Hospital approved this study. All participants provided written informed consent to participate in the study.

One hundred consecutive patients with PD and their spouses/caregivers from our outpatient clinic in the Department of Neurology were included in this study. A diagnosis of PD was made by neurologists according to well-established criteria [16]. Drug-induced parkinsonism, vascular parkinsonian syndrome and atypical parkinsonian syndrome, such as progressive supranuclear palsy and multiple system atrophy, were carefully ruled out by clinical examination and brain imaging. The exclusion criteria were as follows: under 20 years of age; dementia, defined as cognitive dysfunction interfering with activities of daily living; and a diagnosis of or treatment for COVID-19. Disease severity was rated according to the Hoehn and Yahr (HY) stage. The levodopa equivalent dose was calculated based on the conversion factors in a recently published proposal [17].

The participants were asked about changes in stress, physical activity, and body weight after midApril 2020, when a nationwide state of emergency was issued and people were requested to refrain from leaving their homes. The patient global impression of change (PGIC) scale, in which individuals selfrate on a scale from 1 "very much improved" to 7 "very much worse", was applied to PD patients to assess motor symptoms (tremor, rigidity, bradykinesia, gait, and postural stability), sleep, and mood after the start of the COVID-19 pandemic, with reference to their condition prior to that time. Additionally, the caregivers/spouses of the PD patients were asked about the changes in the patients' symptoms that they observed.

We used the short form (SF)-8 Japanese version to assess health-related QOL during the COVID19 pandemic [18]. The SF-8 has 8 subscales to measure physical functioning, role physical, bodily pain, general health, vitality, social functioning, role emotional and mental health. Two summary scores, the physical component summary (PCS) and mental component summary (MCS) scores, were also calculated from the weighted sums of the relevant subscale scores. Subscale and summary scores range from 0 to 100 , with higher values indicating better QOL. The
Hospital Anxiety and Depression Scale (HADS) was applied to evaluate anxiety and depression [19]; it consists of 14 subitems, with 7 for anxiety and 7 for depression, and the scores range from 0 to 21 for each subscale [20]. The HADS has been validated and shown to be useful for application in PD patients, and scores of $>10$ indicate the presence of anxiety or depression [21]. We also collected information on the impact of COVID-19 on hospital visits and interest in and concern about COVID-19.

\section{Statistical analysis}

To compare results between PD patients and their caregivers/spouses, the Mann-Whitney U test or Student's $t$ test was used for continuous variables; chi-square tests were employed for the categorical variables, as appropriate. Stepwise linear regression analyses were performed to determine the healthrelated QOL (MCS and PCS scores on the SF-8) of the $P D$ patients and their caregivers/spouses. Missing values of independent variables were determined by multiple imputation. Results across 10 imputed data sets were combined. Two-tailed $p$ values $<0.05$ were considered to be statistically significant. Because of the exploratory nature of our study, we did not perform adjustments of multiplicity for multiple tests. IBM SPSS Statistics v26.0 (IBM SPSS, Tokyo, Japan) was used for the statistical analyses.

\section{RESULTS}

Table 1 shows the demographics and clinical characteristics of PD patients and their caregivers. PD patients were older and had a lower proportion of smokers than caregivers. The mean evaluation period after the end of the state of emergency was $2.6 \pm 1.7$ months for both groups. Overall, the proportions of individuals with anxiety and depression did not differ between the groups. However, PD patients had a significantly higher rate of comorbidities than caregivers $(63.0 \%$ vs. $42.0 \%, p=0.003)$, though no significant difference in the comorbidity rate between PD patients and caregivers was found, except that diabetes mellitus was more prevalent in the former than the latter $(10.0 \%$ vs $3.0 \%, p=0.045)$ (Supplementary Table 1). Regarding health-related QOL as measured by the SF-8, physical function, role physical, general health, vitality and PCS scores were significantly lower in PD patients than in caregivers. The PGIC scale scores showed the same worsening trends reported by patients and caregivers. 
Table 1

Clinical background, demographics and symptoms associated with COVID-19

\begin{tabular}{|c|c|c|c|}
\hline & $\mathrm{CG}(n=100)$ & $\mathrm{PD}(n=100)$ & $p^{\mathrm{a}}$ \\
\hline$\overline{\text { Age, } y}$ & $65.5 \pm 12.0$ & $72.2 \pm 9.1$ & $<0.001$ \\
\hline $\operatorname{Sex}(M / F)$ & $47 / 53$ & $45 / 55$ & 0.777 \\
\hline Smoking, n (\%) & & & 0.007 \\
\hline Never & $67(67.0)$ & $74(74.0)$ & \\
\hline Past & $21(21.0)$ & $25(25.0)$ & \\
\hline Current & $12(12.0)$ & $1(1.0)$ & \\
\hline \multicolumn{4}{|l|}{ Alcohol intake, n (\%) } \\
\hline Never & $50(50.0)$ & $59(59.0)$ & 0.434 \\
\hline$<1$ day/week & $21(21.0)$ & $23(23.0)$ & \\
\hline 1-2 days/week & $11(11.0)$ & $8(8.0)$ & \\
\hline 3-5 days/week & $7(7.0)$ & $3(3.0)$ & \\
\hline 6-7 days/week & $11(11.0)$ & $7(7.0)$ & \\
\hline Caffeine, n (\%) & $92(92.0)$ & $90(90.0)$ & 0.621 \\
\hline Caffeine, cups/day & $2.7 \pm 1.8$ & $2.3 \pm 1.6$ & 0.098 \\
\hline $\begin{array}{l}\text { Evaluation period after the end of the state } \\
\text { of emergency (days) }\end{array}$ & $79.1 \pm 54.3$ & $79.1 \pm 54.3$ & - \\
\hline Onset age of PD (y) & - & $66.4 \pm 9.6$ & - \\
\hline Disease duration $(\mathrm{y})$ & - & $5.8 \pm 4.4$ & - \\
\hline Hoehn and Yahr stage & - & $2.5 \pm 0.9$ & - \\
\hline $\mathrm{LED}$, mean (median) $\pm \mathrm{SD}, \mathrm{mg} /$ day & - & $479.8(400.0) \pm 308.0$ & - \\
\hline Comorbidities $^{\mathrm{b}}, \mathrm{n}(\%)$ & $42(42.0)$ & $63(63.0)$ & 0.003 \\
\hline Anxiety, n (\%) & $6(6.0)$ & $6(6.0)$ & 1.000 \\
\hline Depression, $\mathrm{n}(\%)$ & $17(17.0)$ & $20(20.0)$ & 0.585 \\
\hline \multicolumn{4}{|l|}{ Health-related QOL (SF-8) } \\
\hline Physical function & $48.3 \pm 6.3$ & $43.9 \pm 7.5$ & $<0.001$ \\
\hline Role physical & $48.4 \pm 6.7$ & $44.3 \pm 8.4$ & $<0.001$ \\
\hline Bodily pain & $47.8 \pm 8.7$ & $45.3 \pm 9.8$ & 0.055 \\
\hline General health & $50.2 \pm 6.9$ & $46.7 \pm 7.2$ & 0.001 \\
\hline Vitality & $50.7 \pm 5.9$ & $47.0 \pm 6.1$ & $<0.001$ \\
\hline Social functioning & $44.6 \pm 9.0$ & $44.5 \pm 8.5$ & 0.949 \\
\hline Role emotional & $47.2 \pm 7.6$ & $46.5 \pm 7.7$ & 0.490 \\
\hline Mental health & $48.9 \pm 6.7$ & $48.6 \pm 6.7$ & 0.804 \\
\hline Physical composite summary & $47.7 \pm 7.0$ & $42.6 \pm 7.2$ & $<0.001$ \\
\hline Mental composite summary & $47.1 \pm 7.2$ & $48.0 \pm 6.9$ & 0.349 \\
\hline
\end{tabular}

Approximately $30-40 \%$ of patients and caregivers reported worsening tremor, rigidity, gait, postural stability, cognition, mood and sleep but not bradykinesia (Fig. 1). These changes in PD-related symptoms, stress, and physical activity showed trends similar to those reported by the two groups. Body weight increased by a smaller percentage in patients than in caregivers (Table 2). Moreover, worsening of PDrelated symptoms, increased stress, and decreased physical activity were observed in $29.0 \%, 37.0 \%$ and $44.0 \%$ of PD patients, respectively. Strong concerns about or interest in COVID-19 were reported by $47.0 \%$ and $69.0 \%$ of caregivers and $50.0 \%$ and $60.0 \%$ of PD patients, respectively. Although 16 patients $(16.0 \%)$ experienced problems with hospital access, none reported medication shortages. Online medical care was used by $9.0 \%$. More than $80 \%$ of the participants wore a mask, but daily mask usage (hours) was significantly shorter in the patient group than in the caregiver group. Regarding determinants of physical (PCS) and mental (MCS) aspects of health-related QOL in PD patients, stepwise linear regression analysis identified that increased gait disturbance and rigidity, HY stage, smoking, LED and decreased body weight predicted worse PCS scores; anxiety, female sex, stress, longer disease duration and depression predicted worse MCS scores (Table 3). In caregivers, age and smoking contributed to worse PCS scores, whereas depression, stress and worsening of patients' mood contributed to worse MCS (Table 4). The results of stepwise linear regression analysis of the subscales of the SF-8 (health-related QOL) are shown in Supplementary Tables 2 and 3. Overall, anxiety, depression, stress, 


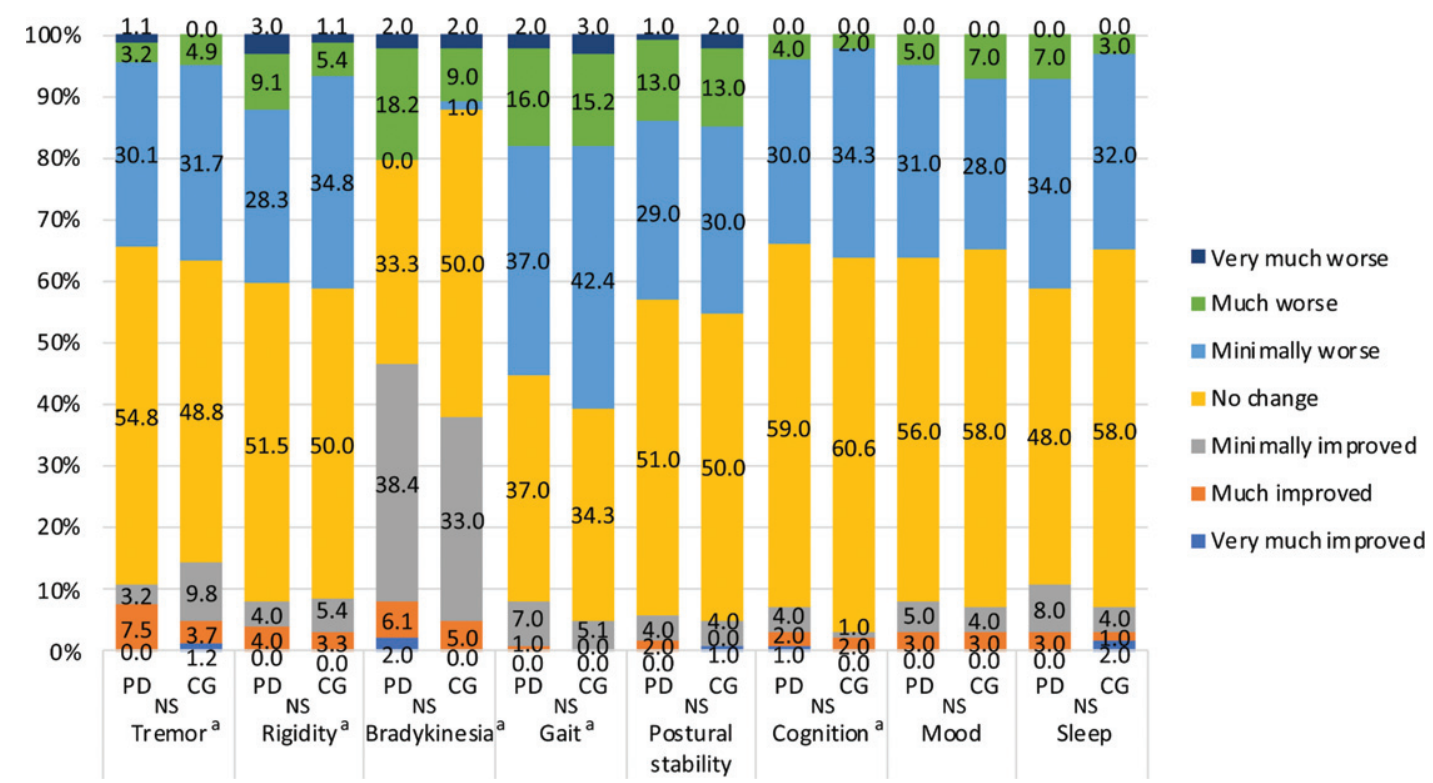

Fig. 1. PGIC scales for tremor, rigidity, bradykinesia, gait, postural stability, cognition, mood and sleep in PD patients and caregivers. ${ }^{*}$ The caregivers assessed the patients' symptoms. PGIC, patient's global impression of change; NS, not significant; PD, Parkinson's disease; $\mathrm{CG}$, caregivers. ${ }^{\mathrm{a}}$ Missing values for tremor $(\mathrm{CG}=18, \mathrm{PD}=7)$, rigidity $(\mathrm{CG}=8, \mathrm{PD}=1)$, bradykinesia $(\mathrm{PD}=1)$, gait $(\mathrm{CG}=1)$, and cognition $(\mathrm{CG}=1)$ were excluded.

and disease severity, as reflected by the HY stage, had negative effects on the subscales in patients with PD. In caregivers, depression and anxiety had negative effects on the subscales. Alcohol consumption had a positive effect on physical function and caffeine intake had a positive effect on bodily pain in the PD patient group.

\section{DISCUSSION}

We found that several subscale scores on the SF8 and physical aspects of health-related QOL (PCS) were significantly more impaired in PD patients than in caregivers. The MCS and PCS scores in our study were significantly lower than the national standard value $(50 \pm 10)$ and comparable to those of Japanese PD patients in nonemergency situations [22]. We also determined key predictors of health-related QOL in patients and caregivers by stepwise linear regression analysis. In our study, a significant number of patients reported worsening of PD-related symptoms, increased stress and decreased physical activity during the COVID-19 pandemic, in agreement with previous studies from other countries [9-12]. KitaniMorii et al. [23] reported that in 71 Japanese PD patients, anxiety and depression were associated with motor experiences of daily living (MDS-UPDRS
II) during the COVID-19 pandemic. Additionally, a study including 38 PD patients and 20 controls showed that during the COVID-19 pandemic, PD patients had more stress, depression, and anxiety, less physical activity, and worse QOL than controls [24].

Increased stress levels during the COVID-19 pandemic are likely to worsen motor symptoms, and reduced physical activity may worsen anxiety, insomnia and constipation [9]. Furthermore, a study evaluating social distancing due to the COVID-19 pandemic found that PD patients who reported being lonely experienced 55\% more severe symptoms over time, and loneliness correlated with decreased QOL [25]. Xia et al. [26] investigated the impact of the COVID-19 pandemic on sleep in patients with PD, with $68.9 \%$ reporting sleep disturbances, as assessed by the Pittsburgh Sleep Quality Index, which was independently associated with the exacerbation of PD symptoms and anxiety. In contrast, changes in sleep status were not a key determinant for health-related QOL in our study; we used the PGIC scale to assess changes in sleep status, which may not have been sufficiently sensitive to detect such changes.

In our study, disease severity, disease duration, gait impairment, rigidity, anxiety, depression and stress were related to the worsening of health-related QOL. According to Fan et al. [27], motor impairment was the most crucial determinant of QOL in PD patients 
Table 2

Changes in symptoms and conditions related to COVID-19

\begin{tabular}{|c|c|c|c|}
\hline & $\begin{array}{c}\text { CG } \\
(n=100)\end{array}$ & $\begin{array}{c}\text { PD } \\
(n=100)\end{array}$ & $p^{\mathrm{a}}$ \\
\hline PD-related symptoms, $\mathrm{n}(\%)^{\mathrm{c}}$ & & & 0.938 \\
\hline Improved & $5(5.0)$ & $4(4.0)$ & \\
\hline Unchanged & $67(67.0)$ & $67(67.0)$ & \\
\hline Worsened & $28(28.0)$ & $29(29.0)$ & \\
\hline Stress, n (\%) & & & 0.192 \\
\hline Decreased & $0(0.0)$ & $1(1.0)$ & \\
\hline Unchanged & $52(52.0)$ & $62(62.0)$ & \\
\hline Increased & $48(48.0)$ & $37(37.0)$ & \\
\hline Physical activity, n (\%) & & & 0.218 \\
\hline Decreased & $34(34.0)$ & $44(44.0)$ & \\
\hline Unchanged & $63(63.0)$ & $51(51.0)$ & \\
\hline Increased & $3(3.0)$ & $5(5.0)$ & \\
\hline Body weight, n (\%) & & & 0.035 \\
\hline Decreased & $14(14.0)$ & $22(22.0)$ & \\
\hline Unchanged & $68(68.0)$ & $71(71.0)$ & \\
\hline Increased & $18(18.0)$ & $7(7.0)$ & \\
\hline Interest in COVID-19, $\mathrm{n}(\%)$ & & & 0.493 \\
\hline Not at all & $0(0.0)$ & $0(0.0)$ & \\
\hline Very little & $1(1.0)$ & $3(3.0)$ & \\
\hline Little & $9(9.0)$ & $12(12.0)$ & \\
\hline Moderate & $21(21.0)$ & $25(25.0)$ & \\
\hline Strong & $69(69.0)$ & $60(60.0)$ & \\
\hline $\begin{array}{l}\text { Concerns about COVID-19, } \\
\mathrm{n}(\%)\end{array}$ & & & 0.770 \\
\hline Not at all & $3(3.0)$ & $1(1.0)$ & \\
\hline Very little & $3(3.0)$ & $5(5.0)$ & \\
\hline Little & $22(22.0)$ & $19(19.0)$ & \\
\hline Moderate & $23(25.0)$ & $25(25.0)$ & \\
\hline Strong & $47(47.0)$ & $50(50.0)$ & \\
\hline $\begin{array}{l}\text { Problems with seeing a } \\
\text { doctor, } \mathrm{n}(\%)\end{array}$ & - & $16(16.0)$ & - \\
\hline $\begin{array}{l}\text { Shortage of medications, } \\
\mathrm{n}(\%)\end{array}$ & - & $0(0.0)$ & - \\
\hline $\begin{array}{l}\text { Received online medical care, } \\
\mathrm{n}(\%)\end{array}$ & - & $9(9.0)$ & - \\
\hline Wore masks, $\mathrm{n}(\%)$ & $92(92.0)$ & $83(83.0)$ & 0.054 \\
\hline Mask usage $^{\mathrm{b}}$ (hours/day) & $4.2 \pm 3.4$ & $2.7 \pm 3.1$ & 0.001 \\
\hline
\end{tabular}

COVID-19, coronavirus disease 2019; CG, caregivers; PD, Parkinson's disease ${ }^{\text {a } U s i n g ~ t h e ~ c h i-s q u a r e ~ t e s t ~ o r ~ S t u d e n t ' s ~} t$ test, values in bold indicate significant differences. ${ }^{b}$ Using the Mann-Whitney $\mathrm{U}$ test after the exclusion of missing values for mask usage in hours per day $(C G=9)$. ${ }^{c}$ The caregivers assessed the patients' symptoms.

without cognitive impairment, whereas depression was the key determinant for QOL in PD patients with cognitive impairment. A systematic review of healthrelated QOL in PD patients identified depression, disease severity and disease disability as determinants [28]. The motor symptoms that most often contribute to QOL include gait impairments, as observed in our study. We also found that female sex was related to worsening of the mental aspects of health-related QOL during the COVID-19 pandemic. Sex differences in motor and several nonmotor features in PD have been explored, and female patients with PD have been found to be more likely to experience anxi- ety, nervousness, sadness, fatigue and constipation [29-31].

In addition to stress and depression, the worsening of patients' mood as perceived by caregivers was a determinant of the mental aspects of the healthrelated QOL of caregivers, suggesting that caregivers were deeply involved in supporting the patients when their motor symptoms deteriorated. Our findings may support a previous study reporting that a greater caregiver burden is associated with a longer disease duration and more motor complications in PD patients [32]. In contrast, the presence of caregivers is associated with higher QOL of PD patients, despite worse cognitive and motor function than those without caregivers [33], indicating the important influence of caregivers on patient outcomes and QOL. We further observed a high rate of agreement between the two groups with regard to changes in patients' symptoms on the PCIG scale during the COVID-19 pandemic, which suggested a high degree of attentiveness on the part of the caregivers. PD patients require multidisciplinary and integrated outpatient care, and receiving that care can improve their healthrelated QOL [34].

The duration of mask use was significantly shorter in the patient group than in the caregiver group, even though there was no difference in the mask use rate. This may reflect the effects of patients' symptoms, such as hypophonia and excessive drooling, which can complicate the prolonged use of masks. Moreover, the increased mask duration among caregivers might be due to their responsibilities, such as shopping, and the ability to go out in a limited capacity into the community compared to PD patients, who may have been more confined to home. In a survey of PD patients during the COVID-19 epidemic, $100 \%$ maintained social distancing, whereas only $37 \%$ wore masks [6].

Virtual visits facilitated by online applications for PD patients are reported to have high feasibility and satisfaction levels [35], and telemedicine approaches should improve care for patients with PD [36]. In an email survey of 1,342 PD patients in the USA, $63.5 \%$ received telehealth services [37]; conversely, only a few patients $(9.3 \%)$ in our study received online medical care. In Japan, telemedicine has been included in universal insurance systems since 2018, but reimbursement for telemedicine by insurance is not sufficient. Nonetheless, a global survey on telemedicine utilization for movement disorders showed that after the onset of the COVID-19 pandemic, the use of telemedicine was increased in 
Table 3

Stepwise linear regression analysis of physical and mental component summary scores of health-related QOL in patients with PD

\begin{tabular}{lcccc}
\hline Variables & $\begin{array}{c}\text { Partial regression } \\
\text { coefficient }\end{array}$ & SE & $95 \%$ CI & $p^{\text {a }}$ \\
\hline Physical component summary & & & & \\
$\quad$ HY stage & -2.256 & 0.724 & -3.675 to -0.837 & 0.002 \\
LED & -3.965 & 1.228 & -6.372 to -1.567 & 0.001 \\
Smoking & -3.004 & 1.282 & -5.516 to -0.492 & 0.019 \\
Body weight & 2.827 & 1.119 & 0.636 to 5.018 & 0.011 \\
Gait & -1.809 & 0.721 & -3.222 to -0.396 & 0.012 \\
$\quad$ Rigidity & -2.167 & 0.738 & -3.615 to -0.720 & 0.003 \\
Mental component summary & & & & \\
Sex & -4.671 & 1.145 & -6.915 to -2.427 & $<0.001$ \\
Disease duration & -0.327 & 0.132 & -0.585 to -0.069 & 0.013 \\
Anxiety & -8.111 & 2.433 & -12.880 to -3.342 & 0.001 \\
Depression & -4.130 & 1.404 & -6.883 to -1.378 & 0.003 \\
Stress & -3.323 & 1.166 & -5.608 to -1.039 & 0.004 \\
\hline
\end{tabular}

QOL, quality of life; PD, Parkinson's disease; SE, standard error; HY, Hoehn and Yahr; LED, levodopa equivalent dose, PGIC, patient's global impression of change; COVID-19, coronavirus disease 2019. ${ }^{\mathrm{a}}$ Using the results from the combined 10 data sets after the application of multiple imputation. Independent variables included in the analysis were age, sex $(M=0, F=1)$, smoking, alcohol, caffeine, disease duration, onset age of PD (years), evaluation period after the end of the state of emergency (days), comorbidity (no $=0$, yes $=1)$, HY stage, LED $(<400 \mathrm{mg} /$ day $=0, \geq 400 \mathrm{mg} /$ day $=1)$, anxiety $($ no $=0$, yes $=1)$, depression (no=0, yes =1), PGIC scale score (1 to 7) for tremor, bradykinesia, rigidity, gait, mood, sleep, cognition, changes in stress (decreased $=0$, unchanged $=1$, increased $=2$ ), physical activity (decreased $=0$, unchanged $=1$, increased $=2$ ), body weight (decreased $=0$, unchanged $=1$, increased $=2$ ), PD-related symptoms (improved $=0$, unchanged $=1$, worsened $=2)$, use of online medical care $($ no $=0$, yes $=1)$, mask usage (hours/day), and interest and concern about COVID-19 (5-point scale).

Table 4

Stepwise linear regression analysis of physical and mental component summary scores of health-related QOL in caregivers

\begin{tabular}{lcccc}
\hline Variables & $\begin{array}{c}\text { Partial regression } \\
\text { coefficient }\end{array}$ & SE & $95 \%$ CI & $p^{\mathrm{a}}$ \\
\hline Physical component summary & & & & \\
$\quad$ Age & -0.179 & 0.055 & -0.286 to -0.072 & 0.001 \\
$\quad$ Smoking & -2.278 & 0.937 & -4.114 to -0.440 & 0.015 \\
Mental component summary & & & & \\
$\quad$ Depression & -5.750 & 1.697 & -9.076 to -2.424 & 0.001 \\
$\quad$ Mood* & -2.065 & 0.833 & -3.697 to -0.432 & 0.013 \\
Stress & -3.773 & 1.292 & -6.306 to -1.240 & 0.004 \\
\hline
\end{tabular}

QOL, quality of life; SE, standard error; PGIC, patient's global impression of change; PD, Parkinson's disease; COVID-19, coronavirus disease 2019. ${ }^{a}$ Using the results from the combined 10 data sets after the application of multiple imputation. Independent variables included in the analysis were age, sex, smoking, alcohol, caffeine, evaluation period after the end of the state of emergency (days), comorbidity (no $=0$, yes $=1$ ), anxiety (no $=0$, yes $=1$ ), depression (no $=0$, yes $=1)$, PGIC scale scores ( 1 to 7 ) for tremor*, bradykinesia*, rigidity*, gait*, cognition*, mood* and sleep*, changes in stress (decreased $=0$, unchanged $=1$, increased $=2$ ), physical activity (decreased $=0$, unchanged $=1$, increased $=2$ ), body weight (decreased $=0$, unchanged $=1$, increased $=2$ ), PD-related symptoms* (improved $=0$, unchanged $=1$, worsened =2), mask usage (hours/day), and interest in and concern about COVID-19 (5-point scale). ${ }^{*}$ The patients' symptoms assessed by the caregivers.

selected regions in Japan [38]. Access to telemedicine depends on ease of availability and varies between countries and regions; however, data regarding how many patients were offered online medical care in this study were not available. Furthermore, medication supply issues may arise during the COVID-19 pandemic [13]. In our study, $16 \%$ of the PD patients reported difficulty in accessing medical facilities. This may be because our university hospital is located in a rural area, where transportation is not as convenient as it is in urban areas, and few patients received alternative treatment options, such as online 
treatment $(9.3 \%)$; regardless, none experienced a medication shortage. In contrast, Guo et al. [39] reported that two-thirds of PD patients had problems receiving medical advice, resulting in a decrease in their QOL.

Our study has several limitations. First, this is a single-center study, and although our region, Tochigi Prefecture, is located in the Kanto region, which includes Tokyo, it is not a representative metropolitan area of Japan. We included caregivers instead of healthy controls in this study, as we sought to ascertain the relationship between caregivers' QOL and PD patient symptoms as perceived by caregivers who are closely involved with these patients. We, however, did not include other family members or home care assistance. Consecutive patients with PD and their caregivers who agreed to participate in the study at our outpatient clinic were included, but the possibility of selection bias cannot be ruled out. In addition, the retrospective nature of this questionnaire-based study, which has no baseline data from the period before the COVID-19 pandemic, may be affected by cognitive impairment and mood, as detailed cognitive tests were not performed and mild cognitive impairment may have been present. Moreover, changes in motor symptoms after the outbreak of COVID-19 were assessed with a questionnaire and not a clinical examination by neurologists, which may result in underestimation and overestimation. Additionally, the nationwide state of emergency is not compulsory, and it is based on the premise of voluntary cooperation by the population. The difference in the precautions taken and the degree of isolation and patient care load might affect mood and caregiver stress, though these aspects were not assessed in our study. We also chose the SF-8 for QOL evaluation instead of the SF-36 because of its shorter administration time in an effort to recruit more patients and caregivers in a limited time period during the pandemic. Because we sought to assess the QOL of patients and caregivers using the same instrument (SF-8), no other PD-specific QOL rating scales, such as the PD Questionnaire-8 or -39, were applied. Finally, the study period of our study was limited to six months, but the impact of recall bias on changes in symptoms should be considered, even though we adjusted for the evaluation period after the COVID-19 pandemic in the statistical analysis.

In conclusion, our study reveals the negative impact of the COVID-19 pandemic on health-related QOL and its determinants in PD patients and their caregivers. The findings of this study may have important implications for the understanding and future management of changes in clinical symptoms of PD patients and their caregivers during the COVID-19 pandemic.

\section{ACKNOWLEDGMENTS}

The authors would like to thank Drs. Yuji Watanabe, Yoshiaki Kaji and Norito Kokubun, Department of Neurology, Dokkyo Medical University, for their assistance with this study. We also appreciate the contributions of Ms. Sanae Tani, Department of Neurology, Dokkyo Medical University, to data entry. Finally, we thank all the participants who agreed to be involved in the study.

This research received no specific grant from any funding agency.

\section{CONFLICT OF INTEREST}

There are no conflicts of interest to report.

\section{SUPPLEMENTARY MATERIAL}

The supplementary material is available in the electronic version of this article: https://dx.doi.org/ 10.3233/JPD-212560.

\section{REFERENCES}

[1] Zhu N, Zhang D, Wang W, Li X, Yang B, Song J, Zhao X, Huang B, Shi W, Lu R, Niu P, Zhan F, Ma X, Wang D, Xu W, Wu G, Gao GF, Tan W, China Novel Coronavirus Investigating and Research Team (2020) A novel coronavirus from patients with pneumonia in China, 2019. N Engl J Med 382, 727-733.

[2] Gualano MR, Lo Moro G, Voglino G, Bert F, Siliquini R (2020) Effects of Covid-19 lockdown on mental health and sleep disturbances in Italy. Int J Environ Res Public Health 17, 4779 .

[3] Schapira AHV, Chaudhuri KR, Jenner P (2017) Non-motor features of Parkinson disease. Nat Rev Neurosci 18, 435450.

[4] Kurisaki R, Ueyama H, Maeda Y, Sakamoto T, Nakahara K, Nakane S, Yamashita S, Ando Y, Kumamoto Earthquake PDSG (2019) Impact of major earthquakes on Parkinson's disease. J Clin Neurosci 61, 130-135.

[5] Cilia R, Bonvegna S, Straccia G, Andreasi NG, Elia AE, Romito LM, Devigili G, Cereda E, Eleopra R (2020) Effects of COVID-19 on Parkinson's disease clinical features: A community-based case-control study. Mov Disord 35, 12871292.

[6] Prasad S, Holla VV, Neeraja K, Surisetti BK, Kamble N, Yadav R, Pal PK (2020) Parkinson's disease and COVID19: Perceptions and implications in patients and caregivers. Mov Disord 35, 912-914. 
[7] Schirinzi T, Cerroni R, Di Lazzaro G, Liguori C, Scalise S, Bovenzi R, Conti M, Garasto E, Mercuri NB, Pierantozzi M, Pisani A, Stefani A (2020) Self-reported needs of patients with Parkinson's disease during COVID-19 emergency in Italy. Neurol Sci 41, 1373-1375.

[8] Salari M, Zali A, Ashrafi F, Etemadifar M, Sharma S, Hajizadeh N, Ashourizadeh H (2020) Incidence of anxiety in Parkinson's disease during the coronavirus disease (COVID-19) pandemic. Mov Disord 35, 1095-1096.

[9] Helmich RC, Bloem BR (2020) The impact of the COVID19 pandemic on Parkinson's disease: Hidden sorrows and emerging opportunities. J Parkinsons Dis 10, 351-354.

[10] Palermo G, Tommasini L, Baldacci F, Del Prete E, Siciliano G, Ceravolo R (2020) Impact of coronavirus disease 2019 pandemic on cognition in Parkinson's disease. Mov Disord 35, 1717-1718.

[11] van der Heide A, Meinders MJ, Bloem BR, Helmich RC (2020) The impact of the COVID-19 pandemic on psychological distress, physical activity, and symptom severity in Parkinson's disease. J Parkinsons Dis 10, 1355-1364.

[12] Brown EG, Chahine LM, Goldman SM, Korell M, Mann E, Kinel DR, Arnedo V, Marek KL, Tanner CM (2020) The effect of the COVID-19 pandemic on people with Parkinson's disease. J Parkinsons Dis 10, 1365-1377.

[13] Papa SM, Brundin P, Fung VSC, Kang UJ, Burn DJ, Colosimo C, Chiang HL, Alcalay RN, Trenkwalder C, MDS-Scientific Issues Committee (2020) Impact of the COVID-19 pandemic on Parkinson's disease and movement disorders. Mov Disord 35, 711-715.

[14] Cubo E, Hassan A, Bloem BR, Mari Z, MDS-Telemedicine Study Group (2020) Implementation of telemedicine for urgent and ongoing healthcare for patients with Parkinson's disease during the COVID-19 pandemic: New expectations for the future. J Parkinsons Dis 10, 911-913.

[15] Antonini A, Leta V, Teo J, Chaudhuri KR (2020) Outcome of Parkinson's disease patients affected by COVID-19. Mov Disord 35, 905-908.

[16] Postuma RB, Berg D, Stern M, Poewe W, Olanow CW, Oertel W, Obeso J, Marek K, Litvan I, Lang AE, Halliday G, Goetz CG, Gasser T, Dubois B, Chan P, Bloem BR, Adler CH, Deuschl G (2015) MDS clinical diagnostic criteria for Parkinson's disease. Mov Disord 30, 1591-1601.

[17] Schade S, Mollenhauer B, Trenkwalder C (2020) Levodopa equivalent dose conversion factors: An updated proposal including opicapone and safinamide. Mov Disord Clin Pract 7, 343-345.

[18] Fukuhara S, Suzukamo Y (2004) Manual of the SF-8 Japanese Version. Institute for Health Outcomes and Process Evaluation Research, Kyoto.

[19] Kitamura T (1993) The Hospital Anxiety and Depression Scale (HADS). Arch Psychiatr Diagn Clin Eval 4, 371-372.

[20] Zigmond AS, Snaith RP (1983) The hospital anxiety and depression scale. Acta Psychiatr Scand 67, 361-370.

[21] Rodriguez-Blazquez C, Frades-Payo B, Forjaz MJ, de Pedro-Cuesta J, Martinez-Martin P, Longitudinal Parkinson's Disease Patient Study Group (2009) Psychometric attributes of the Hospital Anxiety and Depression Scale in Parkinson's disease. Mov Disord 24, 519-525.

[22] Miyashita M, Narita Y, Sakamoto A, Kawada N, Akiyama M, Kayama M, Suzukamo Y, Fukuhara S (2011) Healthrelated quality of life among community-dwelling patients with intractable neurological diseases and their caregivers in Japan. Psychiatry Clin Neurosci 65, 30-38.

[23] Kitani-Morii F, Kasai T, Horiguchi G, Teramukai S, Ohmichi T, Shinomoto M, Fujino Y, Mizuno T (2021)
Risk factors for neuropsychiatric symptoms in patients with Parkinson's disease during COVID-19 pandemic in Japan. PLoS One 16, e0245864.

[24] Shalash A, Roushdy T, Essam M, Fathy M, Dawood NL, Abushady EM, Elrassas H, Helmi A, Hamid E (2020) Mental health, physical activity, and quality of life in Parkinson's disease during COVID-19 pandemic. Mov Disord 35, 10971099.

[25] Subramanian I, Farahnik J, Mischley LK (2020) Synergy of pandemics-social isolation is associated with worsened Parkinson severity and quality of life. NPJ Parkinsons Dis 6, 28.

[26] Xia Y, Kou L, Zhang G, Han C, Hu J, Wan F, Yin S, Sun Y, Wu J, Li Y, Zhang Z, Huang J, Xiong N, Wang T (2020) Investigation on sleep and mental health of patients with Parkinson's disease during the Coronavirus disease 2019 pandemic. Sleep Med 75, 428-433.

[27] Fan Y, Liang X, Han L, Shen Y, Shen B, Chen C, Sun Y, Wang J, Tang Y (2020) Determinants of quality of life according to cognitive status in Parkinson's disease. Front Aging Neurosci 12, 269.

[28] Soh SE, Morris ME, McGinley JL (2011) Determinants of health-related quality of life in Parkinson's disease: A systematic review. Parkinsonism Relat Disord 17, 1-9.

[29] Leentjens AF, Dujardin K, Marsh L, Martinez-Martin P, Richard IH, Starkstein SE (2011) Symptomatology and markers of anxiety disorders in Parkinson's disease: A crosssectional study. Mov Disord 26, 484-492.

[30] Martinez-Martin P, Falup Pecurariu C, Odin P, van Hilten JJ, Antonini A, Rojo-Abuin JM, Borges V, Trenkwalder C, Aarsland D, Brooks DJ, Ray Chaudhuri K (2012) Genderrelated differences in the burden of non-motor symptoms in Parkinson's disease. J Neurol 259, 1639-1647.

[31] Picillo M, Palladino R, Erro R, Alfano R, Colosimo C, Marconi R, Antonini A, Barone P, PRIAMO study group (2021) The PRIAMO study: Age- and sex-related relationship between prodromal constipation and disease phenotype in early Parkinson's disease. J Neurol 268, 448-454.

[32] Tan MMJ, Lim EC, Nadkarni NV, Lye WK, Tan EK, Prakash KM (2019) The characteristics of patients associated with high caregiver burden in Parkinson's disease in Singapore. Front Neurol 10, 561.

[33] Prizer LP, Kluger BM, Sillau S, Katz M, Galifianakis NB, Miyasaki JM (2020) The presence of a caregiver is associated with patient outcomes in patients with Parkinson's disease and atypical parkinsonisms. Parkinsonism Relat Disord 78, 61-65.

[34] Rajan R, Brennan L, Bloem BR, Dahodwala N, Gardner J, Goldman JG, Grimes DA, Iansek R, Kovacs N, McGinley J, Parashos SA, Piemonte MEP, Eggers C (2020) Integrated care in Parkinson's disease: A systematic review and metaanalysis. Mov Disord 35, 1509-1531.

[35] Shalash A, Fathy M, Dawood NL, Hamid E (2020) Adopting virtual visits for Parkinson's disease patients during the COVID-19 pandemic in a developing country. Front Neurol 11, 582613 .

[36] Dorsey ER, Okun MS, Bloem BR (2020) Care, convenience, comfort, confidentiality, and contagion: The $5 \mathrm{C}$ 's that will shape the future of telemedicine. J Parkinsons Dis 10, 893-897.

[37] Feeney MP, Xu Y, Surface M, Shah H, Vanegas-Arroyave N, Chan AK, Delaney E, Przedborski S, Beck JC, Alcalay RN (2021) The impact of COVID-19 and social distancing on people with Parkinson's disease: A survey study. NPJ Parkinsons Dis 7, 10. 
[38] Hassan A, Mari Z, Gatto EM, Cardozo A, Youn J, Okubadejo N, Bajwa JA, Shalash A, Fujioka S, Aldaajani Z, Cubo E, International Telemedicine Study Group (2020) Global survey on telemedicine utilization for movement disorders during the COVID-19 pandemic. Mov Disord 35, 1701-1711.
[39] Guo D, Han B, Lu Y, Lv C, Fang X, Zhang Z, Liu Z, Wang $X$ (2020) Influence of the COVID-19 pandemic on quality of life of patients with Parkinson's disease. Parkinsons Dis 2020, 1216568 . 